\title{
EFECTOS DE LA CRISIS ECONÓNIMA SOBRE LA SALUD MENTAL EN LA POBLACIÓN ESPAÑOLA
}

\section{Economic crisis effects about mental health in Spanish population}

Agar Marín Morales, Lucía Gil Domínguez, Vanesa Román Macías, Luca Farella. Universidad de Granada

El propósito del presente estudio es conocer la prevalencia de personas con problemas de salud mental a causa de la crisis económica, en concreto aquellas que se encuentren en la situación de desempleo o disminución de clase social. Se trata de un estudio transversal en el que participaron 95 sujetos. Las herramientas utilizadas han sido un instrumento de cribado de salud mental General Health Questionnaire (GHQ-28) y el Cuestionario de Ansiedad Estado/Rasgo (STAI). Se realizaron análisis correlaciónales y comparaciones de medias. Entre los resultados obtenidos podemos destacar la relación existente entre la sintomatología depresiva con respecto al descenso de la clase social y la situación laboral. Estos resultados reflejan la necesidad de crear programas preventivos y de intervención sobre dicho problema.

Palabras clave: Crisis económica,Salud Mental, Desempleo, Clase social

The purpose of this study is to know the prevalence of people with mental health problems because of the economic crisis, in particular those that are in the situation of unemployment or decline of social class. This is a cross-sectional study involving 95 subjects. The tools used have been a screening instrument in mental health General Health Questionnaire (GHQ-28) and anxiety Questionnaire State/Trait (STAI). Correlational analyzes were performed and comparisons. Among the results we can emphasize the relationship between depressive symptomatology in relation to the decline of the social class and the labor situation. These results reflect the need to create preventive programs and intervention on this issue.

Key words: Economic crisis, Mental Health, Unemployment, Social class

\section{INTRODUCCIÓN}

Desde 2008, la crisis económica ha afectado de manera considerable en multitud de aspectos individuales, sociales, económicos y culturales. Entre las consecuencias principales de esta situación se encuentran el aumento del desempleo y la bajada de ingresos (Uutela, 2010). En España, en concreto, el Instituto Nacional de Estadística (INE), muestra una tasa de paro en el primer trimestre de 2008 de 2.174 .200 personas (INE, 2008) y en el primer trimestre de 2013 de 6.202.700 personas (INE, 2013). Observando estos datos, se aprecia un considerable incremento que está teniendo graves repercusiones en un gran porcentaje de la población española.El sujeto está influenciado por el contexto en el que vive, y en la actualidad dicho contexto está 
caracterizado por acontecimientos estresantes provocados por la crisis (Uutela, 2010). El desempleo es considerado como uno de los eventos de la vida más estresantes (Buendía, 1990) y tiene un impacto negativo sobre el bienestar psicológico de los empleados (Murphy y Atlanasou, 1999). Estudios muestran que quienes pierden el empleo durante periodos de crisis pueden tener peor estado de salud general que aquellos que lo pierden en otras circunstancias (Brenner y Mooney, 1983; Carlisle, 2008). Otros estudios confirman la existencia de una relación entre el estado socioeconómico y los síntomas depresivos (Mossakowski, 2009). Los síntomas depresivos son las variables más frecuentes en personas desempleadas (Murphy y Atlanasou, 1999), que en casos extremos, y cada vez con más frecuencia, derivan en el suicidio (Uutela, 2010; Gili, Roca, Sanjay, McKee y Stuckler, 2012). El desempleo afecta de forma más acentuada en hombres que en mujeres, esto está relacionado con la cultura, la estigmatización sobre cómolos hombres deben ser los responsables del mantenimiento del hogar (Mossakowski, 2009). Existen discrepancias entre diversas investigaciones en base a la influencia del tiempo de desempleo. Uno de los estudios muestra que el deterioro de la salud mental va aumentando hasta un punto cuyo máximo se sitúa entre los tres y los seis meses, y hacia los seis meses parece estabilizarse el deterioro a medida que la situación se va haciendo más controlable y predecible (Warr, 1987). No obstante, en un estudio realizado por Del Pozo, Ruiz, Pardo y San Martin (2002), los resultados muestran que el deterioro en la salud mental aumenta entre los siete y doce meses y mejora levemente en períodos más largos de desempleo. La bajada de ingresos supone un descenso en la escala social, aunque no está claro que la presencia de trastornos de depresión y ansiedad estén relacionadas con un nivel bajo socioeconómico (Fryers, Melzer y Jenkins, 2003; Laakson en et al., 2007). Sin embargo, los problemas asociados a la bajada de ingresos, y con ello, la bajada en la clase social, son buenos predictores de la aparición de síntomas depresivos (Butterworth et al., 2009; Friedman y Thomas, 2008; Molarius et al., 2009). El estudio de Gili et al. (2012) muestran aumentos significativos respecto al periodo anterior a la crisis en el estado de ánimo, la ansiedad, los trastornos somatomorfos y los relacionados con el alcohol.

Según el marco conceptual propuesto, se considera que se trata de un fenómeno social que está teniendo graves repercusiones en la población española. Por ello, resulta de vital importancia contribuir al estudio científico en dicho tema a fin de sensibilizar a la población en general e intervenir sobre la afectada.

Nuestro estudio pretende verificar las siguientes hipótesis:

1. El estado de ansiedad es mayor en las personas que han descendido de clase social con respecto a las que se han mantenido o han ascendido.

2. En personas que han descendido de clase social, existirán mayores niveles de depresión que las personas que se mantienen.

3. Las personas desempleadas tendrán mayores niveles de depresión que las personas empleadas.

4. Entre las personas desempleadas, se presentan niveles más bajos de salud general percibida en aquellas que llevan entre 7 y 12 meses de desempleo.

5. El desempleo produce mayores niveles de ansiedad en hombres que en mujeres. 


\section{MÉTODO}

\section{Participantes}

Se ha optado por un estudio transversal en el que se ha obtenido una muestra de 95 personas, entre los cuales 44 son hombres y 51 mujeres, con edades comprendidas entre 30 y 60 años $(M=44.34, D T=8.54)$. Todos los participantes de la investigación son de nacionalidad y residencia española.

\section{Instrumentos}

Para llevar a cabo el estudio se han empleado varias herramientas. Para medir el estado de salud general se ha utilizado el General Health Questionnaire (GHQ) de Goldberg (1979) en la versión de 28 ítems (GHQ-28), del cual se ha comprobado la validez a través de varios estudios.La versión española está validada por Lobo (1981, 1986).

Dicho cuestionario se divide en cuatro subescalas: Síntomas somáticos, Ansiedad/Insomnio, Disfunción social y Depresión grave. Cada una de ellas se evalúa a través de siete ítems, contestadas a partir de una escala de tipo Likert.

Por otra parte, se ha utilizado el State-Trait Anxiety Inventory (STAI)(Cuestionario de Ansiedad Estado/Rasgo) de Spielberger, Gorsuch y Lushene (1982). Dicho cuestionario está compuesto por dos subescalas, Estado y Rasgo. Teniendo el objetivo de evaluar el nivel de ansiedad actual de la persona, solo se ha administrado la subescala Estado. Está compuesta por 20 ítems y las respuestas posibles están organizadas en una escala de tipo Likert (0-3).

Además, se han añadido varias cuestiones socioeducativas como la edad, el sexo, el nivel académico, la situación laboral y los cambios referentes a la clase social.

\section{Procedimiento}

La muestra del estudio fue seleccionada mediante muestreo no probabilístico, en concreto el de accesibilidad y el de bola de nieve.

Con el fin de obtener una muestra representativa de la situación en la población española, se ha administrado los diversos instrumentos a sujetos de diferentes comunidades autónomas, como Andalucía, Extremadura, Murcia, Madrid, País Vasco y Cataluña entre los meses de Abril y Mayo de 2013.

\section{Análisis de datos}

Para analizar los datos obtenidos, se ha utilizado el programa estadísticoinformático SPSS 20.0 (IBM SPSS).

Se han realizado análisis descriptivos, comparaciones de medias, análisis de la varianza (ANOVA) y análisis de correlaciones bivariadas. 


\section{RESULTADOS}

Con el objetivo de analizar si existían diferencias entre los participantes en función del mantenimiento o descenso de la clase social en las puntuaciones obtenidas en ansiedad y salud mental en sus diferentes dimensiones, ansiedad-insomnio, depresión grave, y se ha realizado una t de student para muestras independientes.

A continuación, se exponen los resultados más relevantes encontrados tras el análisis de datos (ver Tabla 1).

Tabla 1. Puntuaciones obtenidas por los participantes en las diferentes medidas en función de la clase social

\begin{tabular}{lcccccc}
\hline & Mantiene Clase & \multicolumn{2}{c}{ Desciende clase } & & \\
& Media & $D T$ & Media & $D T$ & $t$ & $p$ \\
\hline DEPRESIÓN GRAVE & .29 & .97 & .78 & 1.18 & -2.17 & .046 \\
\hline ANSIEDAD INSOMNIO & 1.71 & 2.42 & 2.68 & 3.06 & -1.69 & .123 \\
\hline TOTAL ANSIEDAD & 25.77 & 5.31 & 26.81 & 5.12 & -.908 & .366 \\
\hline
\end{tabular}

Como se puede observar en la Tabla 1., no hay diferencias significativas $(p>0.05)$ entre la el total de Ansiedad y el descenso o mantenimiento de la clase social. Sin embargo, sí se encuentran diferencias significativas en relación con la Depresión.

Con el objetivo de analizar la existencia de diferencias según el sexo y la situación laboral (desempleado, empleado) con respecto a los niveles de ansiedad y la sintomatología depresiva se ha realizado una prueba $t$ de student para muestras independientes.

Tabla 2. Puntuaciones obtenidas por los participantes en depresión y ansiedad en función del sexo

\begin{tabular}{lccccccc}
\hline & \multicolumn{3}{c}{ Mujer } & Hombre & & \\
& Media & $D T$ & Media & $D T$ & $t$ & $p$ \\
\hline TOTALANSIEDAD & 26,5 & 5,57 & 25,7 & 4,9 & - & 0,765 & .45 \\
\hline Total Depresón Grave &, 45 & 1,19 &, 45 &, 92 &, 016 & .99 \\
\hline
\end{tabular}

Como se observa en la Tabla 2, no hay diferencias según el sexo con respecto a los niveles de ansiedad y depresión. 
Tabla 3. Puntuaciones obtenidas por los participantes en depresión y ansiedad en función de la situación laboral

\begin{tabular}{lcccccc}
\hline & \multicolumn{2}{c}{ Empleado } & \multicolumn{2}{c}{ Desempleado } & & \\
& Media & DT & Media & $D T$ & $t$ & $p$ \\
\hline TOTALANSIEDAD & 26,5 & 5,017 & 25,45 & 5,7 & 0,91 & .36 \\
\hline Total Depresón Grave &, 26 &, 60 &, 82 & 1,6 & -1.98 & .05 \\
\hline
\end{tabular}

Como se puede observar en la Tabla 3, existen diferencias significativas con respecto a la situación laboral en los niveles de depresión, de modo que los desempleados presentan mayores niveles de depresión en comparación con los empleados. Sin embargo, no se han encontrado diferencias significativas en los niveles de ansiedad.

Con el objetivo de analizar la existencia de relación entre los meses de desempleo y la salud general y la ansiedad, se han realizado correlaciones bivariadas.

Los resultados muestran que no existe una correlación significativa entre los meses de desempleo y los niveles de Ansiedad y Salud general (depresión grave, ansiedadinsomnio, disfunción social y síntomas somáticos). Los resultados han mostrado una correlación positiva y significativa en la relación entre la salud general y la ansiedad ( $r=$ $0.399, p<001)$.

\section{DISCUSIÓN Y CONCLUSIONES}

Según los resultados obtenidos, se puede afirmar lo siguiente: La primera Hipótesis, consistente en que "El estado de ansiedad es mayor en las personas que han descendido de clase social con respecto a las que se han mantenido o han ascendido", se rechaza, ya que no se han encontrado diferencias significativas en la Ansiedad de las personas que se han mantenido en la misma clase y las que han descendido. Cabe mencionar que no se puede afirmar la ausencia de diferencias en los niveles de ansiedad en relación con sujetos que han ascendido, puesto que en nuestra investigación nadie considera que ha subido de clase social.

Con respecto a la segunda Hipótesis, la cual afirmaba que en las personas que han descendido de clase social, existirán mayores niveles de depresión que las personas que se mantienen. Se confirma, puesto que los datos son significativos, y por ello, la disminución de la clase social está relacionada con la aparición de síntomas depresivos.

La tercera Hipótesis,que consiste en“Las personas desempleadas tendrán mayores niveles de depresión que las personas empleadas", se confirma, puesto que existen diferencias significativas según la situación laboral en los niveles de depresión. 
La cuarta Hipótesis, la cual consistía en“Entre las personas desempleadas, se presentarán niveles más bajos de salud general percibida en aquellas que llevan entre 7 y 12 meses de desempleo", se rechaza, puesto que no se ha encontrado una relación significativa.

En relación a la quinta Hipótesis, basada en "El desempleo produce mayores niveles de ansiedad en hombres que en mujeres", se puede decir que se rechaza, puesto que no existen diferencias significativas en cuanto al sexo entre los desempleados y empleados en los niveles de ansiedad.

Con respecto a las limitaciones de nuestro estudio, cabe destacar las siguientes: La metodología utilizada, basada en la encuesta, ha podido producir diversos sesgos, como pueden ser la deseabilidad social, debido ala existencia de ítems demasiado comprometedores para los sujetos y han podido contestar según la norma establecida y no lo que piensen realmente, aun conociendo su anonimato.Por otro lado, ha podido influir en los resultados, el sesgo de la selección de la muestra, a pesar de que se ha intentado que los sujetos fueran representativos de la población, se considera que la muestra debería haber sido mayor y con mayor diversidad de residencia. Además, se debe mencionar la insuficiencia de pruebas para evaluar los efectos de la crisis económica y verificar nuestras hipótesis, a pesar de que la metodología utilizada tenga la ventaja de ser rápida, suponer bajo coste y evitar la fatiga del sujeto.

Como variables extrañas que han podido distorsionar el estudio, se encuentran las situaciones personales de cada individuo, dado que pueden haber reflejado puntuaciones significativas en ansiedad y depresión debido a otros factores que no se han controlado, sin relación directa con la crisis económica.

Con el fin de ampliar el conocimiento sobre los efectos de la crisis económica en la salud mental de la población, se propone llevar a cabo una investigación longitudinal para comprobar el efecto de los meses de desempleo en la salud mental de una persona de forma válida y fiable. Por otra parte, se propone estudiar otras variables relevantes que se han podido modificar a causa de la crisis, como por ejemplo locus de control, autoestima, autoeficacia, apoyo social, etc.

A pesar de la existencia de resultados no significativos, se muestra una relación entre la crisis económica y la salud mental de los sujetos, por lo que se considera conveniente crear programas de intervención para aquellas personas afectadas y programas de prevención para evitar el deterioro de salud mental en el colectivo de riesgo, es decir, aquellos que presenten alta probabilidad de sufrir estas consecuencias. 


\section{REFERENCIAS BIBLIOGRÁFICAS}

Brenner, M. Harvey and Anne Mooney.(1983). Unemployment and Health in the Context of Economic Change.Social Science Medicine, 17 (16), 1125-1138.

Buendía, J. (1990). Psicopatología del desempleo. Anales de psicología, 6(1), 21-36.

Butterworth, P., Rodgers, B., Windsor, T.D. (2009). Financial hardship, socio-economic position and depression: Results from the PATH Through the Life survey. Social Science and Medicine, 69, 229-237.

Carlisle, D. (2008). Public health in a recession.Nursing times, 104(47), 20-23.

Del Pozo, J.A., Ruiz, M.A., Pardo, A. y San Martin, R. (2002). Psicothema, 14(2), 440443.

Friedman, J. y Thomas, D. (2008). Psychological health before, during, and after an economic crisis: results from Indonesia, 1993-2000. World Bank Economic Review, 23, 57-76.

Fryers, T., Melzer, D., y Jenkins, R. (2003). Social inequalities and the common mental disorders: A systematic review of the evidence. Social psychiatric and Psychiatric Epidemiology, 38, 229-237.

Gili, M., Roca, M., Sanjay, B., McKee, y M.,Stuckler, D.(2012).The mental health risks of economic crisis in Spain: Evidence from primary care centres, 2006 and 2010. European Journal of Public Health, 23(1), 103-108.

Goldberg, D.P. y Hillier, V.F. (1979).A scaled version of the General Health Questionnaire, Psychological Medicine, 9, 139-145.

Instituto Nacional de Estadística (2008).Encuesta de Población Activa (EPA): Primer trimestre de 2008.

Recuperado dehttp://www.ine.es/daco/daco42/daco4211/epa0108.pdf

Instituto Nacional de Estadística (2013). Encuesta de Población Activa (EPA): Primer trimestre de 2013.

Recuperado dehttp://www.ine.es/daco/daco42/daco4211/epa0113.pdf

Laaksonen, E., Martikainen, P., Lahelma, E., Lallukka, T., Rahkonen, O., Head, J., et al. (2007). Socioeconomic circumstances and common mental disorders among Finnish and British public sector employees: Evidence from the Helsinki Health Study and the Whitehall II Study. International Journal of Epidemiology, 36, 776-786.

Lobo, A., Pérez-Echeverria, M.J, y Artal, J. (1986).Validity of the scaled versión of the General Health Questionnaire (GHQ-28) in a Spanish population, Psychological Medicine, 16, 135-140. 
Molarius A, Berglund K, Eriksson C, et al. (2009). Mental symptoms in relation to socioeconomic conditions and lifestyle factors: A population based study in Sweden. BMC Public Health, 9, 302.

Mossakowski, K., (2009). The Influence of Past Unemployment Duration on Symptoms of Depression Among Young Women and Men in the United States. American Journal of Public Health, 99(10), 1826-1832.

Murphy, G. y Athanasou, J. (1999). The effect of unemployment on mental health.Journal of Occupational and Organizational Psychology, 72, 83-99.

Spielberger, C.D., Gorsuch, R.L., y Lushene, R. (1982). Manual del Cuestionario de Ansiedad Estado/Rasgo (STAI). Madrid, España: TEA Ediciones.

Uutela, A. (2010). Economic crisis and mental health. CurrentOpinion in Psychiatry, 23, 127-130.

Warr, P.B. (1987). Work, Unemployment and Mental Health. England: Oxford, Scienced Publications. 